\title{
The extraordinary FR II microquasar S26 in NGC
} 7793

Jess W. Broderick, ${ }^{*}$ Manfred W. Pakull, ${ }^{b}$ Roberto Soria, ${ }^{c}$ Stéphane Corbel $^{d}$ and Christian Motch ${ }^{b}$

${ }^{a}$ School of Physics and Astronomy, University of Southampton, Southampton, Hampshire SO17 1BJ, United Kingdom

${ }^{b}$ University of Strasbourg, CNRS UMR 7550, Observatoire Astronomique, 11 rue de l'Université, 67000 Strasbourg, France

${ }^{c}$ Mullard Space Science Laboratory, University College London, Holmbury St. Mary, Surrey RH5 6NT, United Kingdom

${ }^{d}$ Université Paris 7 and Service d'Astrophysique, UMR AIM, CEA Saclay, F-91191, Gif sur Yvette, France

E-mail: J.Broderickesoton.ac.uk manfred.pakulleastro.unistra.fr roberto.soriadmssl.ucl.ac.uk, stephane.corbeldcea.fr, christian.motcheastro.unistra.fr

We have conducted a multi-wavelength study of the recently-discovered microquasar S26 in NGC 7793. This system resembles the famous Galactic microquasar SS 433, but on an even grander scale. A large $(\sim 300 \mathrm{pc} \times 150 \mathrm{pc})$ shock-ionized nebula, detected in $\mathrm{H} \alpha$ and He II $\lambda 4686$, spatially coincides with a synchrotron-emitting radio cocoon. Furthermore, there is a pair of prominent FR II-like radio lobes, an X-ray core with a blue optical counterpart, and a pair of X-ray hotspots that are well-aligned with the radio lobes and X-ray core. Sensitive, highresolution radio observations from the Australia Telescope Compact Array have played a crucial role in our investigation. We give an overview of our results, and discuss how this system offers new insights into the physics of black hole accretion and feedback.

ISKAF2010 Science Meeting

June $10-142010$

Assen, the Netherlands

\footnotetext{
* Speaker.
} 


\section{Introduction}

Microquasars (e.g. overview by [1]), powered by stellar-mass black holes, can be thought of as scaled-down versions of their quasar cousins. One important difference is that, when compared to AGN in dynamical terms, microquasars are mostly located in relatively low-pres sure environments [2]. We therefore expect to see fewer, dimmer cocoons and radio lobes associated with microquasars; however, the linear sizes of these cocoons and jets can be up to 1000 times larger than in radio galaxies and quasars, scaled to their respective black hole masses.

So far, our knowledge of the interaction between microquasar jets and the interstellar medium

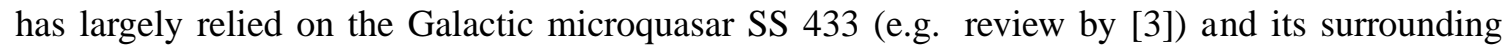
synchrotron-emitting nebula W50 (size $\sim 100 \mathrm{pc} \times 50 \mathrm{pc}$ ), which exhibits 'ear-like' lobes that are inflated by a mildly relativistic, precessing jet. Another ideal laboratory for studying how energy is transferred from powerful jets to the surrounding gas was recently discovered by [П]: the spectacular radio/optical/X-ray microquasar S26 in NGC 7793. Here, we present an overview of some of the results from our ongoing multi-wavelength study of S26; more detailed accounts can be found in both [ [ 4 and [5].

\section{Radio observations}

High-resolution, sensitive radio images of S26 were obtained on 2009 August 6 and 7 with the Australia Telescope Compact Array (ATCA). Simultaneous observations were carried out at 5.5 and $9.0 \mathrm{GHz}$ with the Compact Array Broadband Backend (CABB); the bandwidth at each frequency is about $2 \mathrm{GHz}$. We used the $6 \mathrm{D}$ array configuration, which has minimum and maximum baselines of 77 and $5878 \mathrm{~m}$, respectively. The total integration time on-source was 13.3 hours.

The deconvolved, total-intensity radio maps are shown in Figure 1; in addition, a two-point spectral index map is shown in Figure 2. The source has an FR II-like structure, with two lobes and a surrounding cocoon clearly visible, though no radio core is detected. At $5.5 \mathrm{GHz}$, the integrated flux density is $2.1 \pm 0.1 \mathrm{mJy}$, which corresponds to a radio luminosity about three times that of Cassiopeia A. The average spectral indices in the lobes are $\sim-0.7$ to -0.6 , consistent with optically-thin synchrotron emission. However, the spectral indices are flatter, and in some cases inverted, outside the lobes. This suggests an additional contribution from free-free emission, and perhaps ongoing ejections near the core. Deeper radio observations will be used to test this hypothesis (see Section 6).

\section{X-ray and optical observations}

NGC 7793 was observed with Chandra/ACIS-S3 in 2003 September; the data are available from the public archives. The total exposure time was $48.9 \mathrm{ks}$.

The key feature of S26 in the X-ray band is the aligned triplet of point-like sources (Figure 1), which we interpret as the $\mathrm{X}$-ray core and hotspots (also see [6]). The faint core $\left(\mathrm{L}_{0.3-8 \mathrm{keV}} \approx\right.$ $6 \times 10^{36} \mathrm{erg} \mathrm{s}^{-1}$ ) has a hard spectrum (power-law photon index $\Gamma=1.4 \pm 0.6$ ), consistent with a stellar-mass black hole in the low/hard state. In addition, the core coincides with a blue point-like optical source ( $B \approx 23 \mathrm{mag}$ and $M_{B} \approx-5 \mathrm{mag}$ ) that emits in He II $\lambda 4686$ (Figure B). This object is possibly a Wolf-Rayet donor star (see [円). 

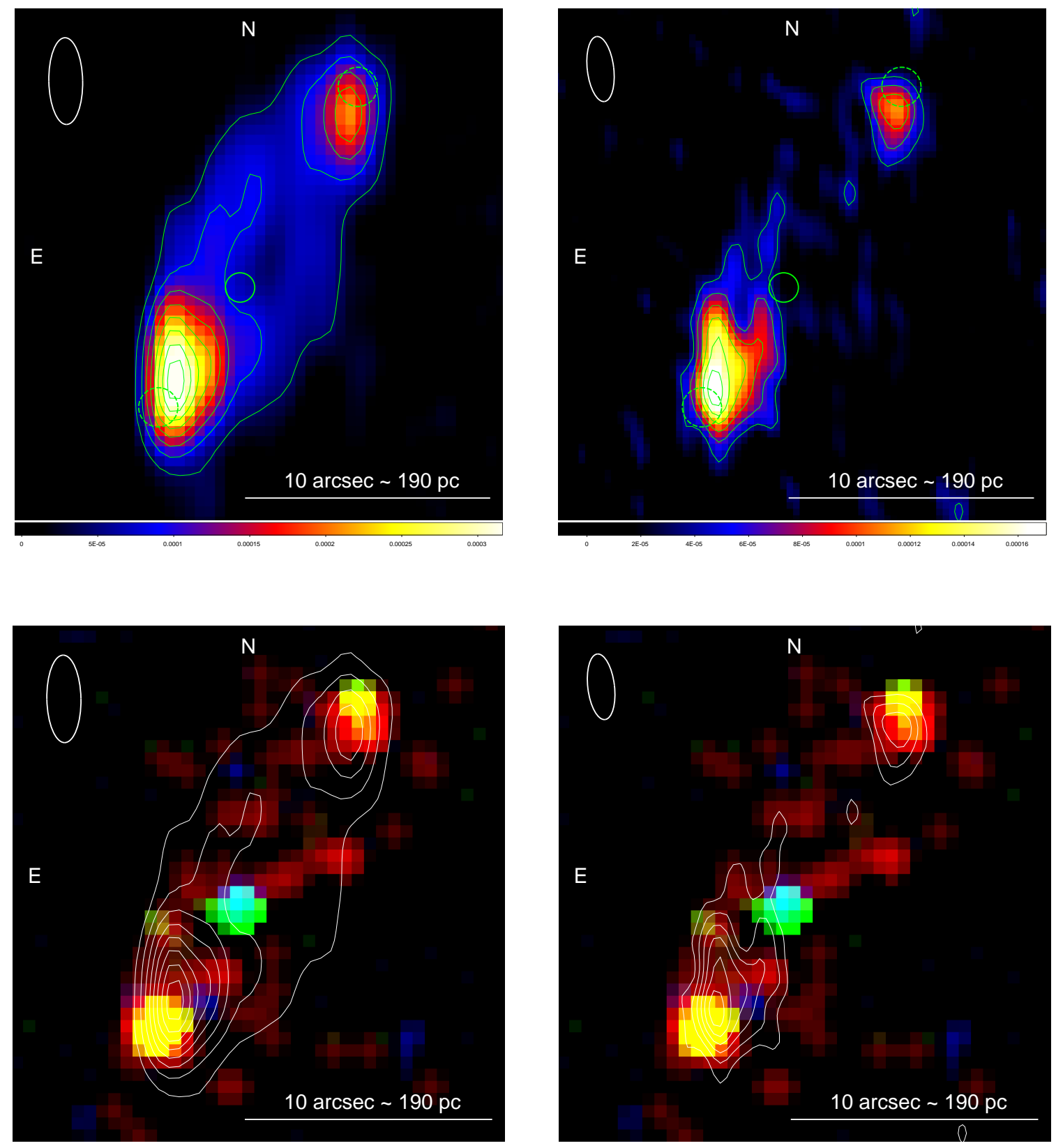

Figure 1: Top two panels: ATCA total-intensity 5.5 and $9.0 \mathrm{GHz}$ images, with intensity scales in Jy beam ${ }^{-1}$. The green circles mark the positions of the X-ray core and hotspots (see bottom two panels; 0.6 arcsec radius for the core and $0.8 \operatorname{arcsec}$ for the hotspots). The angular resolutions are $3.54 \operatorname{arcsec} \times 1.38 \operatorname{arcsec}\left(\mathrm{PA} 1.1^{\circ}\right)$ and $2.67 \operatorname{arcsec} \times 1.08$ arcsec $\left(\mathrm{PA} 6.8^{\circ}\right)$ at 5.5 and $9.0 \mathrm{GHz}$, respectively; the synthesized beam is shown in the top left-hand corner of each panel. At $5.5 \mathrm{GHz}$, the contour levels are 40, 84, 129, 173, 217, 261, 306 and $350 \mu \mathrm{Jy} \mathrm{beam}^{-1}$, while the rms noise level is $8.5 \mu \mathrm{Jy} \mathrm{beam}^{-1}$. At $9.0 \mathrm{GHz}$, the contour levels are 39 , 65, 91, 117, 144 and $170 \mu \mathrm{Jy}_{\text {beam }}{ }^{-1}$ (rms $13.5 \mu \mathrm{Jy}_{\text {beam }}{ }^{-1}$ ). At $5.5 \mathrm{GHz}$, the peak flux densities for the northern and southern lobes are 0.21 and $0.37 \mathrm{mJy} \mathrm{beam}^{-1}$, respectively, while at $9.0 \mathrm{GHz}$ the corresponding measurements in the higher-resolution map are 0.11 and $0.19 \mathrm{mJy}^{\text {beam }}{ }^{-1}$. Bottom two panels: Chandra colour map of S26, smoothed with a 1 arcsec Gaussian core, and overlaid with 5.5 and $9.0 \mathrm{GHz}$ contours. The colour coding is as follows: red, $0.2-1 \mathrm{keV}$; green, $1-2 \mathrm{keV}$; and blue, 2-8 keV. All four panels are from [5]. 


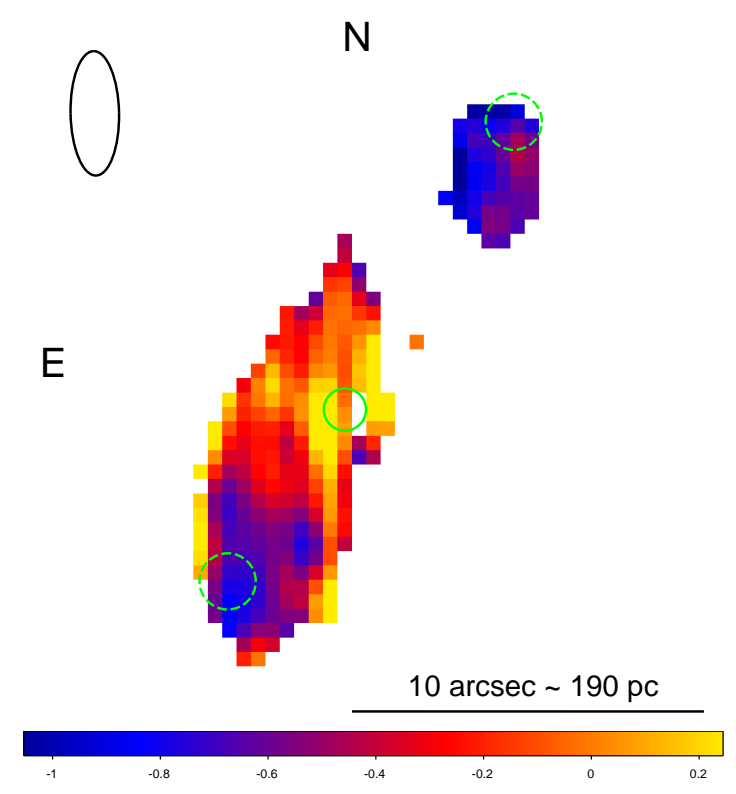

Figure 2: Two-point spectral index map between 5.5 and $9 \mathrm{GHz}$ (where flux density $S_{v} \propto v^{\alpha}$ ). The $9.0 \mathrm{GHz}$ data were tapered to the resolution of the $5.5 \mathrm{GHz}$ data before the map was created. X-ray core and hotspot positions as well as the ATCA synthesized beam are shown as in Figure 1. Figure from [5].

The two hotspots have much softer X-ray spectra. We find that $\mathrm{L}_{0.3-8 \mathrm{keV}} \approx 5 \times 10^{36} \mathrm{erg}$ $\mathrm{s}^{-1}$ and $11 \times 10^{36} \mathrm{erg} \mathrm{s}^{-1}$ for the northern and southern hotspot, respectively (similar to the ratio of radio flux densities). The hotspot spectra are consistent with optically-thin thermal plasma emission from hot, shocked gas, likely located between the reverse and forward shocks. We estimate a hot gas density $\gtrsim 1 \mathrm{~cm}^{-3}$ and a mass $\sim 10^{36} \mathrm{~g}$.

Figures 11 and 3 show that the spatial extents of the $\mathrm{X}$-ray emission, optical nebula (detected in both $\mathrm{H} \alpha$ and $\mathrm{He}$ II $\lambda 4686)$ and radio cocoon are very similar $(\sim 300 \mathrm{pc} \times 150 \mathrm{pc})$. One difference, however, is that each X-ray hotspot appears projected approximately 15-20 pc downstream along the jet direction from the corresponding radio hotspot; the projected distance between the X-ray hotspots is $\approx 290 \mathrm{pc}$, which is about 15 per cent larger than the projected distance between the radio hotspots $(\approx 250 \mathrm{pc})$. The core is along the line of the hotspots, but not symmetrically located between them: it is projected about $110 \mathrm{pc}$ from the southern X-ray hotspot and about $180 \mathrm{pc}$ from the northern X-ray hotspot. This may be due a denser interstellar medium south of the core. Deeper radio observations (Section 6) will allow us to test whether this is the case, such as through the use of Faraday rotation measure synthesis (e.g. [7]).

\section{Energetics of the system}

The total particle energy in the bubble is $\sim 10^{53} \mathrm{erg}$. Both the size and total energy content are comparable to the values found for some ultraluminous X-ray sources (e.g. [8-[10]). Based on the measured radio flux and size of the bubble, and using standard equipartition relations for microquasar lobes, we estimate that the energy carried by the synchrotron-emitting relativistic electrons 

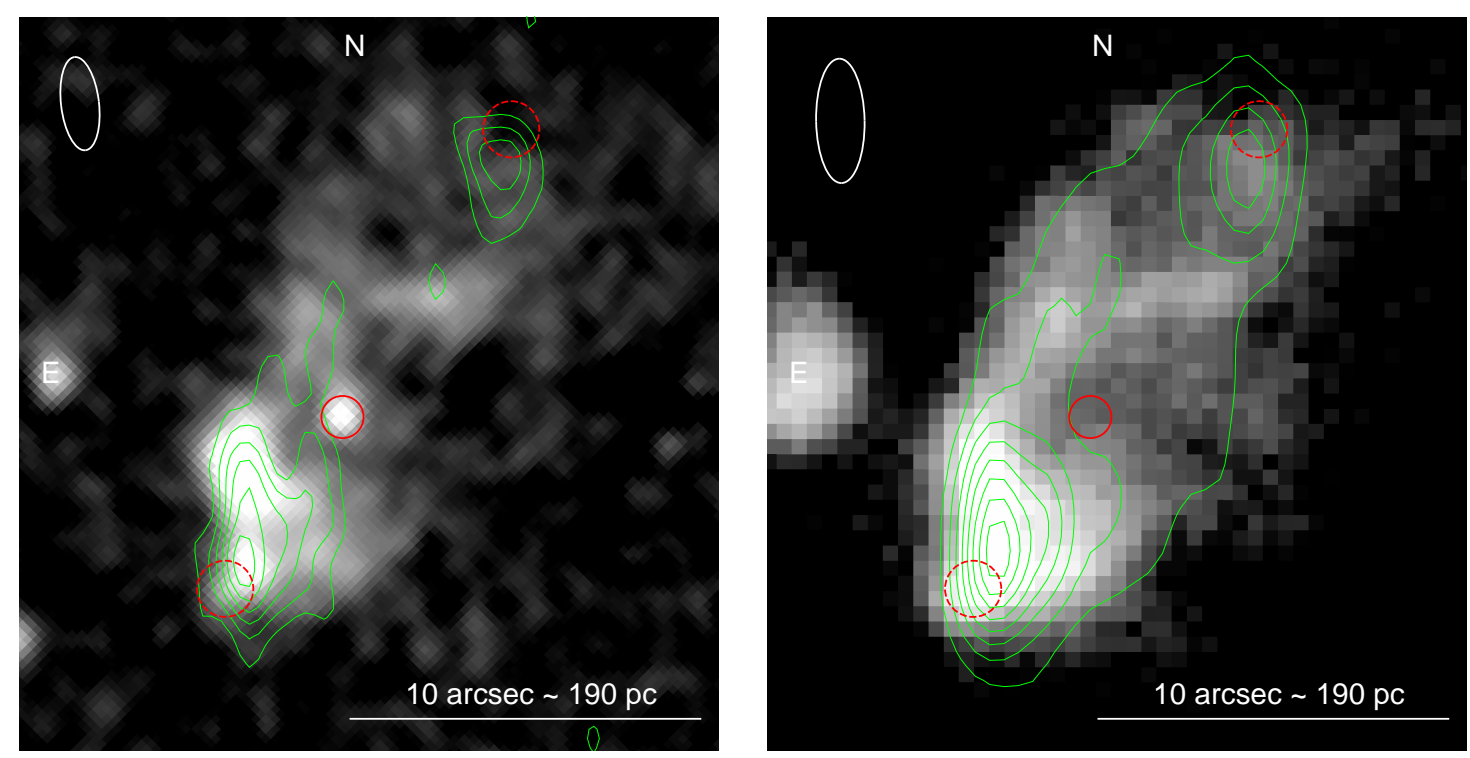

Figure 3: Left: Continuum-subtracted He II $\lambda 4686$ emission, overlaid with ATCA $9.0 \mathrm{GHz}$ contours as in Figure 1. The image was obtained with the VLT/FORS1 in 2002 November; we have smoothed it with a 0.6 arcsec Gaussian core. See [丹] for a discussion of the bright optical core. Right: Continuum-subtracted $\mathrm{H} \alpha$ emission, overlaid with ATCA $5.5 \mathrm{GHz}$ contours. The image was taken with the CTIO $1.5 \mathrm{~m}$ telescope in 2001 October. Both panels are from [5]; X-ray core and hotspot positions as well as the ATCA synthesized beams are shown as in Figure 1.

is a few hundred times less than the energy stored in the protons, nuclei and non-relativistic electrons; non-relativistic particles provide most of the pressure to inflate the bubble. See [5] for the detailed calculations.

\section{What can we learn about black hole accretion and feedback?}

If the black hole in S26 is of stellar origin, its super-Eddington jet power $\left(\sim 10^{40} \mathrm{erg} \mathrm{s}^{-1}\right.$; [4]) may force us to rethink the 'canonical' scheme of black hole accretion states. Though collimated jets are present in Galactic black hole transients when the accretion rate is less than a few percent of the Eddington rate (low/hard state), the jets are quenched at higher accretion rates ( $\sim 0.05-0.5$ Eddington), where the accretion flow usually collapses to a geometrically-thin, radiatively-efficient thermal disk. On the other hand, S26 suggests that steady, collimated jets can exist at superEddington accretion rates.

For S26, the jet power would be severely underestimated if the radio observations were used alone. Yet the radio emission can be the primary diagnostic tool for more distant AGN, where the $\mathrm{X}$-ray and/or optical emission may be much harder to detect. Our observations therefore suggest that some black hole jets could be more powerful than previously thought. This has important consequences for the degree of feedback and heating of the ambient medium, which in turn has a marked effect on the evolution of the host galaxy. 


\section{Future work}

Additional high-resolution ATCA/CABB observations at 2.45, 5.5 and $9.0 \mathrm{GHz}$ were obtained recently on 2010 June $27-29$. The total integration time on-source at $2.45 \mathrm{GHz}$ was 9.9 hours, while the integration time at both 5.5 and $9.0 \mathrm{GHz}$ has now been increased to 33.3 hours. These observations will be used to (i) search for the radio core, (ii) verify the presence of flat-spectrum emission at the bases of the jets, (iii) map the cocoon at $2.45 \mathrm{GHz}$, which will enable us to measure spectral indices over a larger frequency baseline, and (iv) determine the polarimetric properties of the source, including the characteristics of the ambient magnetoionic environment.

\section{Acknowledgments}

JWB acknowledges support from The Science and Technology Facilities Council, UK. RS acknowledges hospitality at Tsing Hua University (Taiwan), at Mount Stromlo Observatory, and at the University of Sydney during the completion of this work. We thank Tasso Tzioumis for his assistance with the ATCA observations. The Australia Telescope is funded by the Commonwealth of Australia for operation as a National Facility managed by CSIRO.

\section{References}

[1] I. F. Mirabel, Microquasars: Summary and Outlook, LNP 794 (2010) 1-15 [astro-ph/0805.2378].

[2] S. Heinz, Radio lobe dynamics and the environment of microquasars, A\&A 388 (2002) L40-L43 [astro-ph/0203295].

[3] S. Fabrika, The jets and supercritical accretion disk in SS433, A\&SPR 12 (2004) 1-152 [astro-ph/0603390].

[4] M. W. Pakull, R. Soria \& C. Motch, A 300-parsec-long jet-inflated bubble around a powerful microquasar in the galaxy NGC 7793, Nature 466 (2010) 209-212.

[5] R. Soria, M. W. Pakull, J. W. Broderick, S. Corbel \& C. Motch, Radio lobes and X-ray hot spots in the microquasar S26, MNRAS (2010) in press.

[6] M. W. Pakull \& F. Grisé, Ultraluminous X-ray Sources: Beambags and Optical Counterparts, in proceedings of A Population Explosion: The Nature \& Evolution of X-ray Binaries in Diverse Environments, AIP Conf. Proc. 1010 (2008) 303-307 [a stro-ph/ 0803 . 4345].

[7] M. A. Brentjens \& A. G. de Bruyn, Faraday rotation measure synthesis, A\&A 441 (2005) 1217-1228 [astro-ph/0507349].

[8] M. W. Pakull \& L. Mirioni, Optical Counterparts of Ultraluminous X-Ray Sources, astro-ph/0202488.

[9] M. W. Pakull \& L. Mirioni, Bubble Nebulae around Ultraluminous X-Ray Sources, in proceedings of Winds, Bubbles, and Explosions: a conference to honor John Dyson, Rev. Mex. Astron. Astrofis. (Ser. Conf.) 15 (2003) 197-199.

[10] M. W. Pakull, F. Grisé \& C. Motch, Ultraluminous X-ray Sources: Bubbles and Optical Counterparts, in proceedings of Populations of High Energy Sources in Galaxies, Proc. IAU Symp. 230 (2006) 293-297 [astro-ph/0603771]. 